\title{
Método de validación estadística del ensayo de respuesta térmica de suelo, serpentín vertical vs horizontal
}

Ing. Valentín Stefanini ${ }^{1}$; Ing. Daniel Nazario ${ }^{2}$

\section{RESUMEN}

Este trabajo se realizó con el objeto de aplicar una comparación estadística entre rectas de calibrado correspondiente a dos Test de Respuestas Térmicas (TRT), sustancialmente diferentes en diseño, lugar y tiempo.

Utilizando un método estadístico de comparación de pendientes de rectas, basado en el análisis de homogeneidad de varianzas.

Primero se realiza un ensayo " $F$ " para determinar si las varianzas son o no homogéneas, y luego un ensayo " $t$ " que determinará la similitud de las pendientes.

De esta manera el ensayo " $F$ ", establece que las varianzas de las pendientes no son homogéneas.

El ensayo " $\mathrm{t}$ " para varianzas no homogéneas, y con probabilidades del 95\%, $97,5 \%$, y $99 \%$ determinando que las pendientes de ambas rectas son diferentes.

E1 mismo ensayo " $t$ " para el 99,5\%, define pendientes similares.
Estableciendo que el método usado en el intercambiador horizontal particular, para obtener el coeficiente de transferencia de calor efectivo de subsuelo es válido.

PALABRAS CLAVE: Comparaciónestadística- test de respuesta térmica.

\section{ABSTRACT:}

This work was carried out in order to apply a statistical comparison between calibration lines corresponding to two Tests of Thermal Responses in situ (TRT), substantially different in design, place and time.

Using a statistical method of comparing straight slopes, based on the analysis of variance bomogeneity.

First an " $F$ "test is carried out to determine if the variances are homogeneous or not, and then a " $t$ "test that will determine the similarity of the slopes.

In this way, the "F" test establishes that the variances of the slopes are not homogeneous.

The " $t$ " test for non-homogeneous variances, with probabilities of $95 \%, 97.5 \%$, and $99 \%$

\footnotetext{
${ }^{1}$ Ingeniero Valentín Stefanini, Universidad Nacional de Villa Mercedes-Argentina. stefanini28@hotmail.com.ar

${ }^{2}$ Ingeniero Daniel Nazario. Universidad Nacional de Villa Mercedes-Argentina. elmundial@hotmail.com
} 
determining that the slopes of both straights are different.

The same " $t$ "test for $99.5 \%$, defines similar slopes.

Establishing that the method used in the particular borizontal exchanger, to obtain the effective coefficient of heat transfer of soil is valid.

KEYWORDS: Comparison - statistics thermal response test.

\section{INTRODUCCIÓN}

El objetivo del presente trabajo es poder validar los resultados obtenidos de un TRT de un intercambiador particular horizontal, respecto de un intercambiador estándar vertical, aplicando un modelo matemático consistente en el proceso de transferencia de calor, con $\lambda$ eff y $\mathrm{Rb}$ como variables de ajuste, desarrollado por Ingersoll, (1948) y Carlslaw y Jaeger (1959), plasmado en la tesis de Eklöf C, Gehlin S. (1996), tomada como referencia.

En San Luis, Argentina, se realizó un ensayo de respuesta térmica (TRT) a un intercambiador particular horizontal, y se lo experimentó aplicando el modelo de la fuente de línea infinita de Kelvin, LSM (Line Source Model) a partir de éste se determinó el coeficiente de conductividad efectivo de subsuelo $\lambda$ eff, y la resistencia térmica de subsuelo $\mathrm{Rb}$, por el método de la pendiente, para la obtención de $\lambda$ eff.

Para saber si los resultados obtenidos en dicho ensayo son fiables, se aplica un método de comparación estadística de pendiente de rectas de calibrado, a las pendientes ob- tenidas en los ensayos de TRT, método propuesto por el autor en el presente trabajo.

La recta de referencia corresponde a un TRT realizado en la Universidad Nacional del Noreste (UNNe) que incumbe a un intercambiador vertical convencional simétrico, que se lo comparó con una recta obtenida de un intercambiador particular horizontal, ensayo realizado en la Universidad Nacional de Villa Mercedes (UNVIME). Éste último fue adecuado a una excavación preexistente para otro fin, quedando empotrado en el medio de un bloque de cemento de $15 \mathrm{~cm}$ de espesor. La parte inferior del bloque está sobre el subsuelo y la parte superior contiene agua de una pileta, de manera que sus características son diferentes, es decir es asimétrico.

La comparación de las pendientes obtenidas por dos ensayos diferentes en tiempo, espacio y configuraciones, nos permite poder validar uno, respecto de otro obtenido por forma estándar.

Este método de validación de pendientes permite:

1.-ESTUDLAR LA REPRODUCIBILIDAD DE UN MÉTODO (Comparar pendientes de rectas obtenidas de momentos diferentes)

2.-ESTUDLAR LA ROBUSTEZ DEL MÉTODO (Comparar pendientes de rectas obtenidas en diferentes condiciones)

3.-COMPROBAR SI LA MUESTRA TIENE EFECTO MATRIZ (Comparar pendientes de rectas calibrado patrón con la de la recta de la muestra) 


\section{METODOLOGÍA}

Para la realización del experimento se utiliza los aportes teóricos de Eklöf $\mathrm{C}$, Gehlin S. (1996), Ingersoll, L.R. and Plass, H.J. 1948 y Carslaw and Jaeger; además se cuenta con los laboratorios de ensayo de la UNVIME.

El experimento que se realizó a partir de ensayos de un intercambiador, es de corte cuantitativo-descriptivo, ya que permite determinar los parámetros del subsuelo y realizar una descripción acerca de cómo el método utilizado durante el experimento podría subsumirse en ahorro de energía debido a la utilización de geotermia de baja entalpía.

Se aplica un ensayo "F" para determinar si las varianzas son o no son homogéneas.

Una vez determinado la homogeneidad de la varianza se procede a realizar un ensayo " $t$ " para determinar la similitud de las pendientes de las rectas.

\section{MATERIALES}

Se utilizó una excavación para la construcción de una piscina de 4,5 $\mathrm{m}$ de largo por 2,3 $\mathrm{m}$ de ancho y de $1,5 \mathrm{~m}$ de profundidad sobre un piso de 7,5 $\mathrm{cm}$ de hormigón. Ya realizado, se colocó un serpentín de caño PEAD 0 3/4" K6 apto para conducciones de agua, se lo sujetó a una malla sima y se lo rellenó con otra capa de hormigón de 7,5 $\mathrm{cm}$ de espesor.

Así, el intercambiador quedó entre un bloque de $15 \mathrm{~cm}$ de hormigón sin ningún tipo de aditivo, como la bentonita. Luego se construyó un tanque de expansión y se lo aisló térmicamente. Se colocó una bom- ba de circulación y luego se instalaron los instrumentos de medida. En el tanque de expansión se colocó una solución de 100 litros de agua con 2 litros de glicol. La perturbación térmica se realizó con una resistencia eléctrica colocada sobre el agua del tanque con su respectiva conexión, también se midió su consumo eléctrico. La medición del caudal se realizó por medio de un caudalímetro mecánico. Posteriormente se determinó que el flujo circulante es turbulento mediante el número de Reynolds.

\section{DESARROLLO}

El objetivo del presente trabajo es poder validar los resultados obtenidos de un TRT de un intercambiador particular horizontal, respecto de un intercambiador estándar vertical, aplicando un modelo matemático desarrollado para este último.

En San Luis, Argentina, se realizó un intercambiador particular horizontal, y se lo ensayo aplicando el modelo de la fuente de línea infinita de Kelvin (LSM), y a partir de este se determina el coeficiente de conductividad efectivo de subsuelo $\lambda$ eff, y la resistencia térmica de suelo $\mathrm{Rb}$, por el método de la pendiente.

Ahora que tan fiables son los resultados obtenidos en dicho ensayo, frente a esta problemática se aplica un método de comparación estadística de pendiente de rectas de calibrado, a las pendientes obtenidas en los ensayos de TRT.

La recta de referencia corresponde aun TRT, realizado en la Universidad Nacional del Noreste (UNNe), el mismo corresponde a un intercambiador vertical estándar, y se 
lo compara con una recta obtenida de un intercambiador particular horizontal, realizado en la Universidad Nacional de Villa Mercedes (UNVIME), esquematizado en la figura 1 , él mismo fue adecuado a una excavación ya existente para otro fin, que- dando empotrado en el medio de un bloque de cemento de $15 \mathrm{~cm}$. de espesor. La parte inferior del bloque esta sobre el subsuelo y la parte superior contiene agua de una pileta, por ello es que sus características son diferentes.

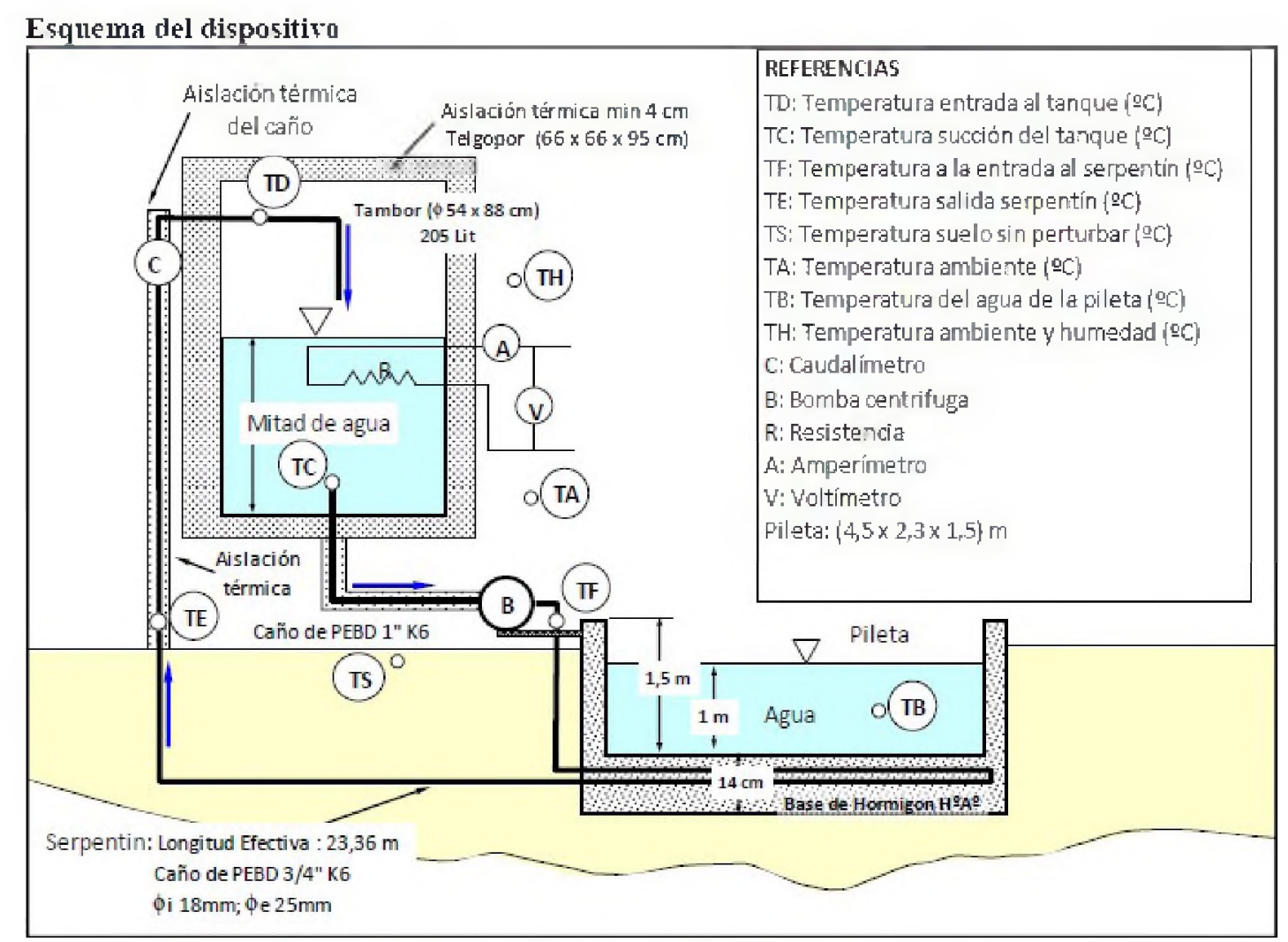

Figma 1. Esquema del dispositivo de medicion montado en San Luis - Argentina.

Las bombas de calor geotérmicas (GSHPs) (ground source heat pumps) son sistemas eficientes para la calefacción y refrigeración de edificios, ampliamente usados en Europa y EE. UU. Estos consisten en acoplar una unidad de bomba de calor y un grupo de intercambiadores de calor de pozo (BHEs) (bore hole heat excharges); Las mejoras que se pueden realizar a estos equipamientos de bomba de calor geotér- mico, están centradas en la optimización del mismo y en la reducción de los costos de instalación; Como estos costos de instalación pueden ser elevados, antes de poner en marcha esta construcción se necesita realizar el cálculo y la simulación para una mayor optimización. Para ello se deben conocer dos parámetros que afectan la eficiencia del depósito de calor, los cuales se deben determinar entre el fluido caloportador y 
la pared del pozo, ellos son: la conductividad térmica efectiva del subsuelo $\lambda$ eff, y la resistencia térmica de subsuelo Rb.. Estos valores se determinaron mediante un Test de Respuesta Térmica (TRT) en situ, que consiste en hacer circular un fluido portador de calor a través de un intercambiador de calor geotérmico.

En el caso de estudio uno vertical estándar de la UNNe y otro particular de la UNVIME.

Estos ensayos in situ se basan en el modelo de la fuente de línea infinita (ILS), de transferencia de calor por conducción térmica de kelvin. Los valores se obtuvieron por el método grafico de la pendiente y posteriormente se realzó un método de validación estadística de los TRT de suelo, ideado por el autor.

Es decir, se va a proceder a aplicar un método estadístico para la comparación de la pendiente de dos rectas de calibrado, basado en el análisis de la homogeneidad de las varianzas.

Las condiciones que deben cumplir los dos ensayos para poder compararlos fehacientemente y determinar si las pendientes de ambos son ó no son estadísticamente similares.

1.- Tener a disponibilidad las bases de datos de los dos ensayos a comparar.

2.- Verificar que las unidades de la variable independiente (eje $\mathrm{x}$ ) sean iguales como también el periodo.

3.- E1 punto de partida y finalización de ambos ensayos deben ser el mismo.

4.- Los números de datos de ambos en- sayos debe ser iguales.

5.- A partir del cumplimiento de los ítems anteriores se obtienen las rectas de regresión lineal de ambos ensayos, como también error medio cuadrático.

6.- Comparación visual del paralelismo de ambas pendientes.

7.- que los errores medios cuadráticos sean parecidos.

Al tener ambas bases de datos nos permite poder determinar las dos rectas de regresión sobre los mismos valores de la variable independiente, en este caso es tiempo, entonces las mismas deben estar dadas en igual unidad, por ejemplo, en segundo. No pudiendo compararse en la variable independiente un ensayo en horas y el otro en segundos. Así mismo si en un ensayo los datos fueron obtenidos cada minuto, y en el otro ensayo cada 10 minutos, se debe unificar el criterio de toma de datos, y corresponde al mayor periodo.

En caso contrario, si el criterio es del menor periodo, la comparación no se puede realizar, por la sencilla razón de que hay incertidumbre en el ensayo de mayor periodo, lo que nos conduce a resultados erróneos.

Por lo expuesto anteriormente entonces los números de datos de ambos ensayos debe ser el mismo o muy parecidos.

Si en la inspección visual del paralelismo de las dos rectas de regresión línea, es significativo el método conduce a que las pendientes no son estadísticamente similares.

Si los errores medios cuadráticos no son parecidos, esto también puede conducir a que las pendientes no sean estadísticamente similares. 
Criterio de tiempo, el modelo matemático utilizado lo establece, es decir que desestima los datos obtenidos por debajo de ese tiempo. En este caso para realizar la comparación se aplica el criterio de tiempo de San Luis el cual resulto de 9 horas. Por lo cual compararemos dos pendientes cuyo punto de inicio es el correspondiente a las 9 horas de ensayo hasta el punto de culminación del ensayo de San Luis el cual fue a las $72 \mathrm{hs.}$

\section{TRT de Corrientes}

El mismo comienza en el tiempo cero hasta el tiempo 426600 segundos, $(118,5$ horas), con una toma de datos por minuto.

\section{TRT de San Luis}

Comienza en el tiempo cero hasta el tiempo 259200 segundos, (72 horas), con una toma de datos cada 10 minuto.

\section{Unificación de criterios}

Por lo expuesto en puntos anteriores, se va a comparar las dos rectas de regresión entre el tiempo 32400 y 259200 segundos correspondientes al TRT SAN LUIS, con criterio de tiempo y en un periodo de $10 \mathrm{mi}-$ nutos, por lo cual se toman del TRT CORRIENTES los datos del mismo periodo.

\section{Recta de regresión lineal del TRT Corrientes}

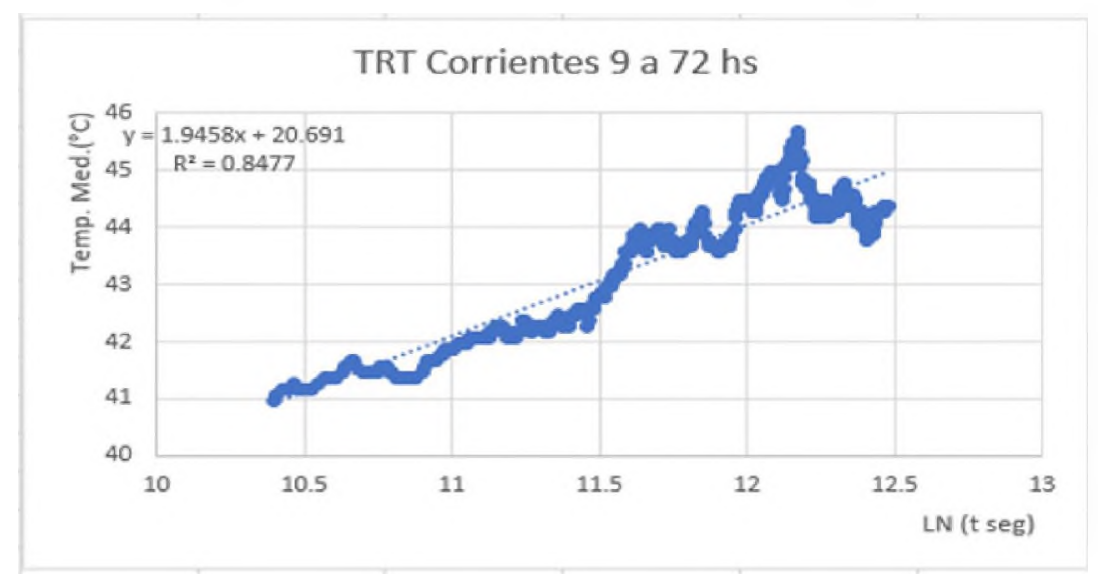

Figura 2: Gráfica del TRT de Corrientes con criterio de tiempo 9 a 72 hs. 
Recta de regresión lineal del TRT Corrientes

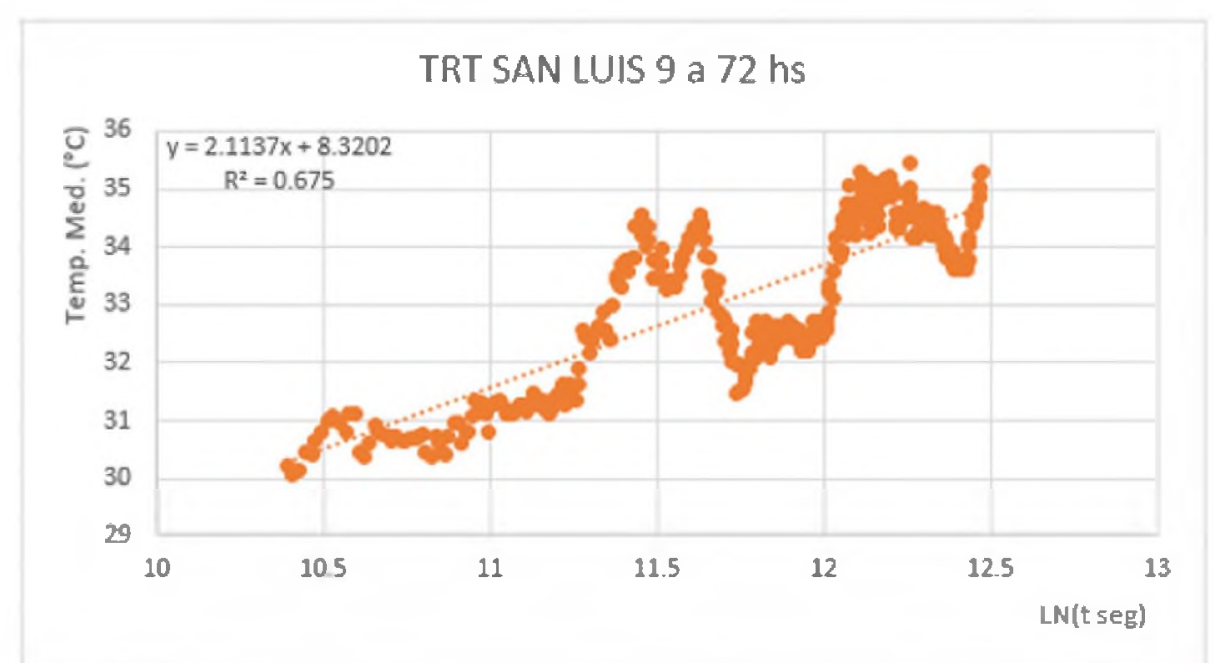

Figura 3: Gráfica del TRT de San Luis con criterio de tiempo 9 a 72 hs.

\section{Datos necesarios para aplicar al ensayo}

En las siguientes tablas se exponen los datos estadísticos de ambos ensayos extraído de la planilla de cálculo.

Estadisticos del TRT Conrientes

\begin{tabular}{|l|l|l|l|}
\hline Pendiente & 1,945774992 & Ordenada & 20,69127688 \\
\hline Sb: Desv. Pend. & 0,042469074 & Sa: Desv. Ord. & 0,500035226 \\
\hline $\mathrm{R}^{\wedge} 2$ & 0,847746375 & Sxy: Desv. Recta & 0,450208327 \\
\hline Grado Libertad & 377 & $\left.\sum\{\text { YY-Y(est.) }]^{\wedge} 2\right\}$ & 76,41320177 \\
\hline \multicolumn{3}{|c|}{ Tabla 1: Datos estadisticos del TRT Corrientes } \\
\hline
\end{tabular}

Ecuación de la recta de regresión lineal del TRT Corrientes

$$
Y(\text { est.Corr })=[(1,95 \pm 0,042) * X+(20,7 \pm 0,5)]
$$

Estadisticos del TRT San Luis

\begin{tabular}{|l|l|l|l|}
\hline Pendiente & 2,11367936 & Ordenada & 8,32021568 \\
\hline Sb: Desv. Pend. & 0,07553806 & Sa: Desv. Ord. & 0,8893928 \\
\hline $\mathrm{R}^{\wedge} 2$ & 0,67499235 & Sxy: Desv. Recta & 0,80076768 \\
\hline Grado Libertad & 377 & $\sum\left\{\left[\mathrm{Y}-\mathrm{Y}(\text { est.) }]^{\wedge} 2\right\}\right.$ & 241,743285 \\
\hline \multicolumn{4}{|l|}{ Tabla 2: Datos estadisticos del TRT San Luis } \\
\hline
\end{tabular}

Ecuación de la recta de regresión lineal del TRT San Luis

$$
Y(\text { est. } S L)=[(2,11 \pm 0,075) * X+(8,32 \pm 0,88)]
$$




\section{Resumen de datos}

Pendientes de las dos rectas

\begin{tabular}{|l|l|}
\hline Bcorr $=1,95$ & Bsl=2,11 \\
\hline
\end{tabular}

Tabla 3: Pendientes

Desviación estándar de las pendientes

\begin{tabular}{|c|c|}
\hline$S_{\text {Bcorr }}=0,042$ & $S_{\text {BSl }}=0,075$ \\
\hline
\end{tabular}

Tabla 4: Desviación estándar de las pendientes

Varianza de las dos pendientes

\begin{tabular}{|c|c|}
\hline$S_{\text {Bcorr }}^{2}=0,0018$ & $S_{\text {Bsl }}^{2}=0,0057$ \\
\hline
\end{tabular}

Tabla 5: Varianza de las pendientes

Números de datos de cada recta:

\begin{tabular}{|l|l|}
\hline Ncorr $=379$ datos & Nsl $=379$ datos \\
\hline
\end{tabular}

Tabla 6: Nimero de datos a considerar

\section{Nota}

Se toman la cantidad de datos de San Luis, los cuales son 379, tomados cada 10 minutos, a partir de las 9 horas de comenzado el ensayo hasta cumplir con las 72 horas propuestas.

\section{Aplicación del estadístico al $95 \%$ de probabilidad}

Determinación si la varianza de las dos pendientes es o no homogéneas, mediante un ensayo " $F$ "

A.- Calculo del estadístico de "F", que lo denominaremos Fcalc.

$$
\begin{gathered}
F_{\text {calc. }}=\frac{\text { VarianzadeSanLuis }}{\text { VarianzadeCorriente }} \\
F_{\text {calc. }}=\frac{S_{\mathrm{Bsl}}^{2}}{S_{\mathrm{Bcorr}}^{2}} \quad F_{\text {calc. }}=\frac{0,0057}{0,0018}=3,16
\end{gathered}
$$

Nota

Fcalc. Siempre debe ser mayor que 1 (uno), esto es lo que condiciona cual varianza va en el numerador y cual en el denominador. En este caso la varianza de San Luis es mayor que la de Corriente. 
Calculo de los grados de libertada del numerador y del denominador

$$
U_{\text {num } / \text { den }}=N-2
$$

Grado de libertad del numerador

$$
\begin{gathered}
U_{\text {numerador }}=N_{s l}-2=379-2=377 \\
U_{s L}=377
\end{gathered}
$$

Grado de libertad del denominador

$$
\begin{gathered}
U_{\text {denominador }}=N_{\text {corr }}-2=379-2=377 \\
U_{\text {corr }}=377
\end{gathered}
$$

Determinación del valor de F tabulado Ftab. 95\%, Usl, Ucorr

El valor de Ftab al $95 \%$ de probabilidad, con grados de libertad del numerador (377) y grados de libertad del denominador (377). En una tabla "F".

Ftab al $95 \%, 377,377$

Eje "x" SL, Eje "y" Corr

Con uso del software "R", obtenemos el valor de tabla F $95,377,377$

$$
\begin{gathered}
F_{.95,377,377}=1.184 \\
F_{\text {calc. }}(3,16)>F_{t a b .}(1,184)
\end{gathered}
$$

Es decir que las varianzas NO SON HOMOGENEAS

Nota

En caso contrario donde Fcalculado sea MENOR que Ftabulado, las varianzas SON HOMOGENEAS.

Deducir si las pendientes son estadísticamente similares con un ensayo "t". PARA VARIANZAS NO HOMOGENEAS AL 95\%

Se realiza un ensayo "t" para varianzas NO HOMOGENEAS. 
Calculo del estadístico tcalc.

$$
\begin{gathered}
t_{\text {calc. }}=\frac{\left|B_{\text {sl. }}-B_{\text {corr },}\right|}{\sqrt{S_{\text {sl. }}^{2}+S_{\text {corr. }}^{2}}} \\
t_{\text {calc }}=\frac{|2,11-1,95|}{\sqrt{0,0057+0,0018}}=2,73 \\
t_{\text {calc }}=2,73
\end{gathered}
$$

Calculo del estadístico t"calc..

$$
t_{c a l c .}^{\prime \prime}=\frac{t_{s l} * S_{\mathrm{Bsl}}^{2}+t_{\text {corr }} * S_{\mathrm{Bcorr}}^{2}}{S_{\mathrm{Bsl}}^{2}+S_{\mathrm{Bcorr}}^{2}}
$$

Dónde: tsl. y tcorr. Se obtienen por medio de tabla.

Calculo de ttab., sl

$$
t_{t a b, 95 \%, N s l-2 g l}
$$

Calculo del grado de libertad San Luis

$$
\begin{gathered}
\text { Gradodelibertad }=N_{\text {sl. }}-2 \\
\text { Gradodelibertad }=379-2=377
\end{gathered}
$$

Con uso del software "R", obtenemos el valor de tabla $t_{.95,377}$ para San Luis

$$
t_{95,377} \simeq 1,645
$$


Calculo de ttab.,com.

$$
t_{t a b, 95 \%, N \operatorname{corr},-2 g l}
$$

Calculo del grado de libertad Corrientes

$$
\begin{gathered}
\text { Gradodelibertad }=N_{\text {corr }}-2 \\
\text { Gradodelibertad }=379-2=377
\end{gathered}
$$

De la tabla "t" o del software "R", obtenemos para Corrientes, para una probabilidad del $95 \%$ y con un grado de libertad de 377

$$
t_{95,377} \equiv 1,645
$$

Calculo del estadístico t'calc.

$$
\begin{gathered}
t_{\text {calc. }}^{\prime \prime}=\frac{1,645 * 0,0057+1,645 * 0,0018}{0,0057+0,0018}=1,62 \\
t_{\text {calc. }}^{\prime \prime}=1,62 \\
t_{\text {calc. }}=2,73 \\
t_{\text {calc. }}=2,73>t_{\text {calc. }}^{\prime \prime}=1,62
\end{gathered}
$$

Es decir, las pendientes son diferentes para el $95 \%$

\section{Aplicación del estadístico al 97,5\% de probabilidad}

Deducir si las pendientes son estadísticamente similares con un ensayo "t". PARA VARIANZAS NO HOMOGENEAS AL 97,5\%

$$
\begin{array}{cl}
\text { Ttab para San Luis } & t_{.975,377}=1.966276 \\
\text { Ttab para Corrientes } & t_{.975,377}=1.966276
\end{array}
$$

$$
\begin{gathered}
t_{\text {calc. }}^{\prime \prime}=\frac{1.966 * 0,0059+1.966 * 0,0018}{0,0059+0,0018}=1,97 \\
t_{\text {calc. }}=2,73>t_{\text {calc. }}^{\prime \prime}=1,97
\end{gathered}
$$

Las pendientes son estadísticamente diferentes para el $97,5 \%$, siendo sus varianzas no homogéneas 


\section{Aplicación del estadístico al $99 \%$ de probabilidad}

Deducir si las pendientes son estadísticamente similares con un ensayo "t". PARA VARIANZAS NO HOMOGENEAS AL $99 \%$

Ttab para San Luis

Ttab para Corrientes

$$
\begin{aligned}
& t_{.99,377}=2,33628 \\
& t_{99,377}=2,33628
\end{aligned}
$$

$$
\begin{gathered}
t_{\text {calc. }}^{\prime \prime}=\frac{2,336 * 0,0059+2,336 * 0,0018}{0,0059+0,0018}=2,34 \\
t_{\text {calc. }}=2,73>t_{\text {calc. }}^{\prime \prime}=2,34
\end{gathered}
$$

Es decir, las pendientes son diferentes para el 99\%, siendo sus varianzas no homogéneas

\section{Aplicación del estadístico al 99,75\% de probabilidad}

El cálculo del $F_{\text {tab. }}$ Al 99,7\% con 377 grados de libertad tanto en el numerador como en el denominador respectivamente es

$$
\begin{gathered}
F_{.9975,377,377}=1.33 \\
F_{\text {calc. }}(3,16)>F_{\text {tab. }}(1,33)
\end{gathered}
$$

Es decir que las varianzas siguen siendo NO HOMOGENEAS

Deducir si las pendientes son estadísticamente similares con un ensayo " $"$ ". PARA VARIANZAS NO HOMOGENEAS AL 99,75\%

Ttab para San Luis

$t_{.9975,377}=2,823$

Ttab para Corrientes

$t_{9975,377}=2,823$

$$
\begin{gathered}
t_{\text {calc. }}^{\prime \prime}=\frac{2,823 * 0,0059+2,823 * 0,0018}{0,0059+0,0018}=2,82 \\
t_{\text {calc. }}=2,73<t_{\text {calc. }}^{\prime \prime}=2,82
\end{gathered}
$$

Las pendientes son estadísticamente similares al 99,75\%, siendo sus varianzas no homogéneas 


\section{DISCUSIÓN}

Mediante un ensayo " $t$ " se pudo determinar que las pendientes son estadísticamente similares al $99,75 \%$ y no así para el $95 \%, 97,5 \%$ y al $99 \%$. Mediante el ensayo "F" se determinó las varianzas en todo el rango propuesto son no homogéneas.

\section{CONCLUSIÓN}

A partir del método aplicado en el TRT San Luis, se puede afirmar que para una certeza del 99,5\% las pendientes de las rectas de San Luis y de Corrientes son similares, por lo tanto, la aplicación del método es correcto.

Con esta nueva perspectiva de comparación de los TRT, dada una excavación se le puede adecuar un intercambiador de calor geotérmico, de diseños diversos. Posteriormente con un TRT de realización rápida y económica, determinamos las $\mathrm{ca}^{-}$ racterísticas del subsuelo. A partir de aquí se realiza la comparación estadística propuesta que validaría dichos parámetros. Si esta validación es correcta se diseña el emprendimiento geotérmico sobre valores reales, lo cual aseguraría una climatización eficiente, generando mayor prestigio de la geotermia de baja entalpía.

\section{REFERENCIAS}

TRT: intercambiador particular horizontal

LSM: Line Source Model

\section{AGRADECIMIENTOS}

A la Dra. Sogari Noemi y al Licenciado Cabral Guillermo, por su desinteresada colaboración proporcionandome los datos del TRT Corrientes. Eternamente agradecido.

\section{BIBLIOGRAFÍA}

Eklöf C, Gehlin S. (1996). A Mobile Equipment for Thermal Response Test. Master of science thesis 1996:198E. Lulea University of Technology. Sweden.

Ingersoll, L.R. and Plass, H.J. 1948." Theory of the ground pipe heat source for the heat pump." Heating, Piping \& Air Conditioning 20/7, pp.119-122

Carslaw and Jaeger, Conduction of Heat in Solids (Oxford science publications, 1964)

Meseguer Lloret Susana 2017. Comparación estadística de rectas de calibrado. Universidad Politécnica de València - UPV

\section{PAGINA WEB.}

https://www.youtube.com/ watch?v=zbf3b8ELP5Y\&t=23s 28/03/2018 